\title{
Blick in den Mund verrät Diabetes
}

— Sie untersuchten 601 Patienten ab 30 Jahren, die eine Zahnklinik in New York aufsuchten. Bei keinem von ihnen lag war bisher die Diagnose Diabetes oder Prädiabetes gestellt worden.

535 Patienten berichteten über einen Risikofaktor für Diabetes, wie positive Familienanamnese, einen hohen Cholesterinspiegel, Bluthochdruck oder Übergewicht. Bei diesen Patienten wurde das Zahnfleisch genau in Augenschein genommen und der Nüchternblutzucker bestimmt.

Hatten die Probanden $\geq 26 \%$ tiefe Zahnfleischtaschen und $\geq 4$ fehlenden Zähnen, lag in $73 \%$ der Fälle ein Diabetes vor. Ein $\mathrm{HbA}_{1 \mathrm{C}}$-Wert von $\geq 5,7 \%$ steigerte die Diabeteswahrscheinlichkeit auf $92 \%$.

\section{Kommentar}

Die Entzündung der Gingiva ist eine frühe Komplikation des Diabetes. Tiefe Zahnfleischtaschen sind ein Diabetessymptom. Zahnmediziner könnten deshalb mit hoher Wahrscheinlichkeit einen Prädiabetes oder Diabetes identifizieren und den betroffenen Patienten eine weitere Untersuchung etwa beim Hausarzt ans Herz legen.

K. MALBERG
- E. Lalla et al.

(Division of Periodontics, Section of Oral and Diagnostic Sciences, College of Dental Medicine, Columbia University, 630 W. 168th Street, PH7E-110, New York, NY 10032, USA; E-mail: EL94@columbia.edu) Identification of Unrecognized Diabetes and Pre-diabetes in a Dental Setting. J. Dent. Res. 90 (2011) 855-860

\section{Diabetes und Depression: wie hängt das zusammen?}

\begin{abstract}
Es wird immer wieder diskutiert, ob Menschen mit Diabetesrisiko durch die Insulinresistenz häufiger depressiv werden oder ob Depression insulinresistent macht. In beiden Fällen besteht eine Koinzidenz, die letztendlich zur Erhöhung des Diabetesrisikos und zur Manifestation eines Diabetes mellitus führen kann.
\end{abstract}

— In der hier vorgestellten Studie sollte der Zusammenhang zwischen gestörter Glukosetoleranz bzw. Typ-2-Diabetes und depressiven Symptomen untersucht werden. Diese Fragestellung ist interessant, da als Bestandteil von Präventionsstrategien in erster Linie eine Lebensstilund Verhaltensänderung im Vordergrund steht. Diese ist umso schwerer zu erreichen, je stärker eine Depression bei der Risikoperson vorliegt.

2712 Teilnehmer der FIND2D-Studie wurden in Finnland zu einem Gesundheitscheck eingeladen. Die depressive Stimmungslage der Teilnehmer wurde mit dem Beck Depression Inventory bestimmt. Alle Personen machten einen oralen Glukosetoleranztest.
Depressive Symptome zeigten 14,4\% der Personen mit normaler Glukosetoleranz, 13,7\% der Probanden mit gestörter Glukosetoleranz, 14,8\% der neu diagnostizierten Diabetiker und 26,4\% der Probanden, bei denen die Stoffwechselstörung schon lange bekannt war.

\footnotetext{
- P. Mantyselka et al.

Association of depressive symptoms with impaired glucose regulation, screen-detected, and previously known type 2 diabetes: findings from the Finnish D2D Survey. Diabetes Care 34 (2011) 71-76
}

\section{Kommentar}

Die Studie macht es nicht einfacher. Gibt es nun einen Zusammenhang zwischen Diabetes und Depression oder nicht? Dieser populationsbasierte Survey zeigt, dass es einen Zusammenhang zwischen bestehendem Diabetes und Depression gibt, der aber durch viele Einflussfaktoren verwischt oder überdeckt ist. Man könnte das Ergebnis dahingehend interpretieren, dass Diabetes per se nicht depressiv macht, aber die Diagnose eines Diabetes einen Patienten depressiv werden lassen kann. Im Zusammenhang mit anderen Studien zu diesem Thema scheint es einen deutlichen Link zu geben zwischen bestehendem Diabetes und depressiven Symptomen. Diese Studie zeigt, dass das erst nach Diagnose relevant wird. Damit ergeben sich neue Ansatzpunkte in der Diabetesprävention und -therapie. Dies wirft unweigerlich die Frage auf, ob die Diagnose des Diabetes beim Patienten aufgrund kognitiver Verarbeitungsprozesse zu einem höheren Depressionsrisiko führt, oder ob die zugrunde liegende Insulinresistenz der entscheidende Trigger ist. Man kann schlussfolgern, dass die Diagnose eines Diabetes den Patienten einem deutlich höheren Risiko aussetzt, depressiv zu werden, und wir das in der Schulung und Intervention bei Diabetespatienten und auch bei Risikopersonen unbedingt im Fokus haben müssen.

P. SCHWARZ = 